variously as volume change, shape change or a result of other processes altering voxel intensity, we dispute the simplistic assertion that region of interest methodologies are 'more accurate' - such methodologies have their own difficulties, in particular with interrater reliability and the optimal parcellation boundaries chosen for structures, and the two methodologies are perhaps better viewed as complementary. Region of interest analyses of a similar sample demonstrated that volume deficits of the hippocampus and amygdala characterise schizophrenia but not bipolar disorder (Marshall et al, 2004; McDonald et al, 2006). This is consistent with our computational morphometry study - and with Kraepelin's seminal dichotomy.

Craddock, N. \& Owen, M. J. (2005) The beginning of the end for the Kraepelinian dichotomy. British journal of Psychiatry, 186, 364-366.

Marshall, N., McDonald, C., Schulze, K., et al (2004) Amygdala volume in patients with schizophrenia or bipolar disorder from multiply affected families and their unaffected relatives. Schizophrenia Research, 67 (suppl. I), 235

McDonald, C., Bullmore, E.T., Sham, P. C., et al (2004) Association of genetic risks for schizophrenia and bipolar disorder with specific and generic brain structural endophenotypes. Archives of General Psychiatry, 61, 974-984.

McDonald, C., Marshall, N., Sham, P., et al (2006) Regional brain morphometry in patients with schizophrenia or bipolar disorder and their unaffected relatives. American Journal of Psychiatry, in press.

Strakowski, S. M., Adler, C. M. \& DelBello, M. P. (2002) Volumetric MRI studies of mood disorders: do they distinguish unipolar and bipolar disorder? Bipolar Disorders, 4, 80-88.

C. McDonald Department of Psychiatry, National University of Ireland Galway, Galway, Ireland. E-mail: colmmcdonald@nuigalway.ie

E. Bullmore Brain Mapping Unit, University of Cambridge, Department of Psychiatry,

Addenbrooke's Hospital, Cambridge, UK

R. Murray Division of Psychological Medicine, Institute of Psychiatry, King's College London, UK

\section{Social defeat and schizophrenia}

Selton \& Cantor-Graae (2005) relate schizophrenia to social defeat. Given Darwin's theory of intrasexual selection, social defeat is inevitable for a proportion of any population, and it is not unlikely that we are seeing this unselected or deselected portion in the psychiatric clinic. The response to social defeat is variable. In chimpanzees there is conditional reconciliation, in which the defeated animal engages in affiliative behaviour with the one who has defeated him (Aureli et al, 2002). The hugging and kissing ritual relieves post-conflict anxiety (indicated by scratching and other selfdirected acts), so that in the chimpanzee world the sun goes down on no one's wrath. In partially migratory species of birds, such as the robin, the defeated birds who have no territories migrate, and if they return in the spring they may find that the winners have succumbed to the cold. In partially hibernating species the defeated animals hibernate. In general, in territorial species defeated animals disperse, whereas in group-living species they stay in the group in a subordinate role.

I think that defeated humans have the alternative defeat strategies of either dispersing or staying in the group. The 'schizotype' appears to be a dispersal phenotype, modified from the usual mammalian dispersal phenotype because of the uniquely cohesive structure of human groups, which are held together by common belief systems. When a person with this phenotype is defeated, they develop a new belief system, recruit followers and take them off to a new location (Stevens $\&$ Price, 2000). This appetitive behaviour may well require stimulation of the dopamine reward system, as was found in defeated mice, which being territorial disperse when defeated. However, when defeated the depression-prone human stays in the group in a subordinate role. He may be happily reconciled to this subordination or he may use the depressive strategy of 'deceiving downwards' in which he develops the cognition that he is not such a useful member of the group as he thought he was (Hartung, 1987). This depressive strategy may involve some downregulation in the hippocampus, as occurs in defeated rats, which are groupliving animals (McEwen, 2005).

In general, we think people with the schizotypal phenotype become depressed when dispersal is blocked whereas those who are prone to depression become depressed when reconciliation is blocked. People with the schizotypal phenotype and depression also have their new belief system, which in the absence of followers is likely to be labelled delusion, and the unworldly prophet is then looked after not by adoring acolytes but by psychiatric nurses.

Aureli, F., Cords, M. \& Van Schaik, C. P. (2002) Conflict resolution following aggression in gregarious animals: a predictive framework. Animal Behaviour, 64 325-343.

Hartung, J. (1987) Deceiving down: conjectures on the management of subordinate status. In Self-Deceit:
An Adaptive Strategy (eds J. Lockard \& D. Pulhus), pp. 170-185. Englewood Cliffs, NJ: Prentice-Hall.

McEwen, B. S. (2005) Glucocorticoids, depression, and mood disorders: structural remodelling in the brain. Metabolism, 54, (suppl. I), 20-23.

Selten, J. P. \& Cantor-Graae, E. (2005) Social defeat: risk factor for schizophrenia? British Journal of Psychiatry, 187, $101-102$.

Stevens, A. \& Price, J. (2002) Prophets, Cults and Madness. London: Duckworth.

J. S. Price South Downs Health NHS Trust, Brighton General Hospital, Elm Grove, Brighton BN2 3EW,UK. E-mail: johnscottprice@hotmail.com

Selton \& Cantor-Graae (2005) proposed that long-term experiences of social defeat may sensitise the mesolimbic dopamine system, increasing the risk for schizophrenia. Regrettably they continued the tradition of ignoring the distal evolutionary perspective. An underemphasised observation is that although neurological illnesses have lifetime prevalence rates in the order of thousands, prevalence rates for psychiatric illness often lie between $1 \%$ (as for schizophrenia) and about $20 \%$. When considering highly disabling conditions such as schizophrenia, depression or anxiety, one must consider the survival implications. Over evolutionary time if there were not some adaptive advantage these genes would have been eliminated. The suggestion that these conditions are products of modern culture is untenable, as they are found in all cultures and have been observed back in time as far as history permits. Furthermore, animals certainly have depression and anxiety.

Selton \& Cantor-Graae could have referred to the book by Stevens \& Price (2000) on the evolutionary adaptiveness of social subordination and schizophrenia. They proposed that schizotypal individuals at times of social crises may come to the fore and lead individuals with similar genes in new directions. Similarly, work by Gilbert (1992) and Sloman (2000) on depression and defeat warrant consideration.

Evolutionary perspectives often suggest obvious but new directions for geneenvironment research. For example, I have proposed a model of post-traumatic stress disorder (PTSD) based on mammalian defences (Cantor, 2005). An understanding of these suggests that looking for genes for the entity PTSD is misguided. The six mammalian defences operate under different selection regimes, therefore greater evolution of one will be associated with a 
decreased need for others. The tortoise, although not a mammal, provides the perfect example: its extraordinary defensive shell reduces the need to flee or fight to virtually zero.

Furthermore, the possibility arises that future gene therapies may have serious untoward consequences (Watson \& Andrews, 2002). The genes associated with social defeat may encourage a battered wife to yield to her brutal husband, albeit at the price of chronic depression. Should her relevant genes be deleted by future gene therapy, potential consequences include being killed as a result of not yielding, killing her abuser in retaliation or at least a heightened state of domestic abuse. The potential for such untoward consequences must be considered.

Cantor, C. (2005) Evolution and Posttraumatic Stress: Disorders of Vigilance and Defence. Hove: Routledge.

Gilbert, P. (1992) Depression: The Evolution of Powerlessness. Hove: Lawrence Erlbaum.

Selton, J. P. \& Cantor-Graae, E. (2005) Social defeat risk factor for schizophrenia? British journal of Psychiatry, 187, $101-102$

Sloman, L. (2000) How the involuntary defeat strategy relates to depression. In Subordination and Defeat: An Evolutionary Approach to Mood Disorders and Their Therapy (eds L. Sloman \& P. Gilbert), pp. 47-67. Mahwah, NJ: Lawrence Erlbaum.

Stevens, A. \& Price, J. (2000) Prophets, Cults and Madness. London: Duckworth.

Watson, P. J. \& Andrews, P.W. (2002) Toward a revised revolutionary adaptationist analysis of depression: the social navigation hypothesis. Journal of Affective Disorders, 72, $1-14$

C. Cantor Department of Psychiatry, University of Queensland 4072, Australia.

E-mail: cantor98@powerup.com.au

Authors' reply: We thank Drs Price and Cantor for their interesting reactions. We welcome the prophet as a much more colourful 'endophenotype' for schizophrenia than a dysfunction in eye-tracking or prepulse inhibition. However, whether the increased reproductive fitness of a small number of prophets compensates for the reproductive impairments in patients with schizophrenia and their lonely schizotypal relatives remains to be demonstrated.

E. Cantor-Graae Department of Community Medicine, Lund University, University Hospital UMAS, Malmö, Sweden

J.-P. Selten Department of Psychiatry, University Hospital, PO Box 85500, 3508 GA Utrecht,

The Netherlands. E-mail: jp.selten@azu.nl

\section{Schizophrenia, cancer and imprinting: early nutritional influences}

We read with interest the important findings of Goldacre et al (2005) on the association between schizophrenia and cancer morbidity. Compared with the general population, they found a reduced rate of cancer of the colon in the schizophrenia cohort (adjusted rate ratio $0.72,95 \% \mathrm{CI}$ $0.50-1.01)$ with a trend towards significance $(P=0.06)$. Rates of rectal cancer were significantly reduced in people with schizophrenia (rate ratio $0.57,95 \% \mathrm{CI}$ 0.33-0.93, $P=0.03)$. In their discussion, they emphasised the reduced rate of skin cancer in the schizophrenia cohort (rate ratio $0.56,95 \%$ CI $0.36-0.83, P=0.004$ ).

Recent studies suggest that abnormal insulin-like growth factor-2 (IGF-2) imprinting is aetiological in the development of colorectal cancer (Jirtle, 2004). Genomic imprinting occurs following epigenetic modification of the germ line, which results in parent-of-origin dependent, monoallelic gene expression in somatic cells. Epigenetic changes in the genome are stable but reversible alterations in a $\mathrm{CpG}$ dinucleotide or histones, for example through changes in methylation. The genome of colonic epithelium from patients with colorectal cancer is hypomethylated compared with normal colonic epithelia (Feinberg \& Vogelstein, 1983). Hypomethylation results in the loss of IGF-2 imprinting. We proposed abnormal imprinting (deletion of paternally expressed IGF-2) as a possible mechanism associated with schizophrenia risk (Abel, 2004). Early nutritional influences (prenatal/maternal) may stimulate changes in cytosine methylation to which imprinted genes such as IGF-2 seem susceptible. Early nutrition may influence susceptibility not only to adult obesity, diabetes and cardiovascular disease (Waterland \& Jirtle, 2004) but also to schizophrenia. This suggests that early nutritional interventions aimed at preventing chronic disease are an exciting possibility in schizophrenia. This view is supported by Dutch and more recent Chinese data which indicated that rates of schizophrenia doubled following prenatal exposure to famine (St Clair et al, 2005).

Abel, K. M. (2004) Foetal origins of schizophrenia: testable hypotheses of genetic and environmental influences. British Journal of Psychiatry, 184, 383-385.

\section{Feinberg, A. P. \& Vogelstein, B. (1983)}

Hypomethylation distinguishes genes of some human cancers from their normal counterparts. Nature, 30I 89-92.
Goldacre, M. J., Kurina, L. M., Wotton, C. J., et al (2005) Schizophrenia and cancer: an epidemiological study. British Journal of Psychiatry, 187, 334-338.

Jirtle, R. L. (2004) IGF2 loss of imprinting: a potentia heritable risk factor for colorectal cancer. Gastroenterology, 126, I190-120I.

St Clair, D., Xu, M., Wang, P., et al (2005) Rates of adult schizophrenia following prenatal exposure to the Chinese famine of 1959-1961. JAMA, 294, 557-562.

Waterland, R. A. \& Jirtle, R. L. (2004) Early nutrition, epigenetic changes at transposons and imprinted genes, and enhanced susceptibility to adult chronic diseases. Nutrition, 20, 63-68.

K. M. Abel Centre for Women's Mental Health Research, University of Manchester, Oxford Road, Manchester MI3 9PL, UK.

E-mail: Kathryn.M.Abel@manchester.ac.uk

M. P. Allin Division of Psychological Medicine, Institute of Psychiatry, London, UK

R. L. Jirtle Department of Radiation Oncology, Duke University, Durham, North Carolina, USA

\section{Post-traumatic stress disorder after life events}

In their interesting article Mol et al (2005), who conclude that life events might cause as many post-traumatic stress disorder (PTSD) symptoms as do traumatic events. The design and conclusions of the study raise some doubts.

Our first concern relates to the assumption that a particular symptom or disorder may be diagnosed in the absence of the fundamental nature or essence of the disorder. The authors claim to identify PTSD in the absence of (a) a traumatic event and (b) a response involving intense fear, helplessness or horror. Both these criteria are diagnostic A criteria of the DSM-IV and essential features of PTSD. Diagnosing PTSD with disregard for a traumatic event is like diagnosing a major depressive disorder in the absence of depressed mood.

Our assumption regarding the broad use of the PTSD diagnosis is strengthened by the instrument used for assessing PTSD symptoms, which has high sensitivity but lacks specificity (Carlson, 1997). In addition, it is questionable whether a self-report scale can assess the clinical relevance of symptoms. Our concern is corroborated by the results in Table 4 . The only item on which the life events group scored higher than the traumatic events group was the non-specific symptom of 'having trouble concentrating', whereas the traumatic events group scored higher on 'trauma-specific items' such as amnesia and hyperarousal. 